\title{
An accurate theoretical description of fluids composed of fully anisotropic molecules: Application to $\mathrm{C}_{2 v}$ symmetry
}

\author{
M. Alvarez, F. Lado, ${ }^{\text {a) }}$ and E. Lomba \\ Instituto de Química Física Rocasolano, CSIC, Serrano 119, E-28006 Madrid, Spain \\ M. Lombardero and C. Martín \\ Instituto de Química Física Rocasolano, CSIC, Serrano 119, E-28006 Madrid, Spain \\ and Departamento de Química Física I, Universidad Complutense, E-28040 Madrid, Spain
}

(Received 17 March 1997; accepted 19 June 1997)

\begin{abstract}
We use two molecular integral equation approximations to compute the thermodynamic properties and microscopic structure of two liquids composed of planar molecules with $\mathrm{C}_{2 v}$ symmetry, namely $\mathrm{SO}_{2}$ and $\mathrm{H}_{2} \mathrm{~S}$. These approximations couple the exact molecular Ornstein-Zernike equation with the hypernetted chain (HNC) and reference-hypernetted chain (RHNC) closures. The theoretical results obtained for various thermodynamic states agree remarkably well with molecular dynamics calculations. In particular, the atom-atom distribution functions are very well reproduced. We find that the RHNC approximation with a hard-sphere fluid reference system offers notable improvement over $\mathrm{HNC}$ in the pressure calculation. We include also a self-consistent mean field calculation to incorporate the effect of polarizability on the dielectric constant of liquid $\mathrm{SO}_{2}$. Final results for this quantity are in excellent agreement with experimental values. In contrast, the model used for the electrostatic interactions in $\mathrm{H}_{2} \mathrm{~S}$ leads to anomalously high permanent dipole moments, compared to experiment, and consequently to dielectric constants that are completely off the experimental data. (C) 1997 American Institute of Physics. [S0021-9606(97)50936-6]
\end{abstract}

\section{INTRODUCTION}

In a recent paper, Lado et al. ${ }^{1}$ presented an algorithm for the numerical solution of Ornstein-Zernike (OZ) integral equations for fluids composed of fully anisotropic particles. As an illustration of the method, they solved the hypernetted chain (HNC) equation for a simple model of liquid $\mathrm{SO}_{2}$ proposed by Sokolic et al., ${ }^{2}$ whereby sulphur dioxide molecules are represented by a three-center Lennard-Jones potential with $\mathrm{C}_{2 v}$ symmetry without electrostatic interactions.

The results of Ref. 1, although taken at relatively high temperature and moderate density, clearly suggested that the proposed algorithm and the HNC approximation are able to produce a reasonable description of not-so-simple fluids. It was also clear, however, that further studies were needed to obtain a better assessment of this approach. This is consequently the main goal of the present work. On the one hand, we seek here to examine thermodynamic states that are more representative of the liquid phase (i.e., lower temperatures and higher densities) and more realistic potential models that include electrostatic interactions. For this, we have focused again on the model used in Ref. 1 together with a point charge model also proposed by Sokolic et al. ${ }^{2}$ On the other hand, we also consider a somewhat less anisotropic system proposed by Forester et al. ${ }^{3}$ to model a different molecular liquid, namely $\mathrm{H}_{2} \mathrm{~S}$. In this case, we use a three-site point charge potential with a single Lennard-Jones center. For these models, we have performed calculations on the liquid side of the phase diagram in the vicinity of the zero-pressure line, which we know to be a good low-temperature estimate

\footnotetext{
a) On sabbatical leave from Dept. of Physics, North Carolina State University, Raleigh, NC 27695.
}

of the liquid branch of the gas-liquid equilibrium curve. It is shown in this work that, good as they seem, the HNC results leave room for improvement, in particular with regard to the pressure, which is typically overestimated. We have thus carried out reference-hypernetted chain (RHNC) ${ }^{4}$ calculations with a hard-sphere reference fluid whose sphere diameter is optimized according to the free energy minimization criterion. ${ }^{5}$ A significant improvement is thereby achieved, especially for the less anisotropic of the two substances, $\mathrm{H}_{2} \mathrm{~S}$.

Comparison with simulation results of the spherical harmonic projections of the molecular pair distribution function calculated in Ref. 1 showed a surprising ability of the HNC approximation to describe the averaged microscopic ordering of the fully anisotropic molecules. However, this type of representation, beyond a few low-order coefficients, has a difficult physical interpretation. A more intuitive picture of the microstructure is often provided by atom-atom distribution functions. Thus another task we undertake here is a straightforward extension of the algorithm proposed by Alvarez et al., ${ }^{6}$ in the context of fluids composed of linear molecules, to calculate the atom-atom pair distribution functions by multidimensional integration over the full molecular pair distribution function. We find that, except for some fine details, both the HNC and the RHNC approximations accurately reproduce the simulated atom-atom correlations.

Finally, an important quantity that can be evaluated from the microscopic information provided by the integral equation calculations is the dielectric constant. Comparison with dielectric constants from simulation, however, is plagued with the numerous uncertainties associated with the determination of this quantity by simulation, which typically requires extremely long runs ${ }^{7}$ and is very sensitive to system 
size. Encouraged by previous experience for different substances like $\mathrm{HCl}$ (Ref. 8) and $\mathrm{CH}_{3} \mathrm{CN}$ (Ref. 9), we decided instead to compare our results with purely experimental data. In order to do this, the effect of polarizability has to be taken into account. This is done using the self-consistent mean field approach (SCMF) proposed by Carnie and Patey. ${ }^{10} \mathrm{We}$ find that the results obtained in this way for liquid $\mathrm{SO}_{2}$, both in HNC and RHNC approximation, agree well with the experimental dielectric constants. In contrast, we conclude that the manner in which the electrostatic interactions are modeled in $\mathrm{H}_{2} \mathrm{~S}$, ${ }^{3}$ while leading to reasonable thermodynamic properties, produces an anomalously high dipole moment which in turn is responsible for excessively high values of the dielectric constant obtained, even without inclusion of polarizability effects. Aside from these modeling problems, the theory performs remarkably well for all the thermodynamic properties and states considered.

The remainder of the paper proceeds along the following lines. The next section offers a brief summary of the elements of the theory required for the calculations carried out in this work. The models and thermodynamic states on which we have focused are presented in Sec. III. In Sec. IV we summarize the main results and conclusions.

\section{A BRIEF SKETCH OF THE THEORY}

An essential part of the calculations presented in this paper consists of the $\mathrm{OZ}$ equation for molecular fluids,

$$
\gamma(12)=\frac{\rho}{8 \pi^{2}} \int d \mathbf{3} c(13)[c(32)+\gamma(32)],
$$

where $\rho$ is the number density and $\int d \mathbf{3}=\int d \mathbf{r}_{3} d \omega_{3}$ denotes integration over particle positions and orientations. This equation forms a closed set together with a general closure relation resulting from a diagrammatic analysis which relates the structure of the liquid and the pair potential $u(12)$,

$$
c(12)=\exp [-\beta u(12)+\gamma(12)+B(12)]-\gamma(12)-1,
$$

where $c(12)$ is the direct correlation function and $\gamma(12)=g(12)-1-c(12)$ is the indirect correlation function, with $g(12)$ the molecular pair distribution function. $B(12)$ is the so-called bridge function, which formally sums a series of complex terms in a diagrammatic density expansion. Here we will consider two approximations to this quantity: $B(12)=0$, the hypernetted chain approximation, and $B(12)=B_{\mathrm{HS}}(r ; d)$, the reference-hypernetted chain approximation, ${ }^{4}$ where $B_{\mathrm{HS}}$ is the bridge function of a reference hard-sphere fluid, calculated with the Verlet-Weis-Grudke-Henderson ${ }^{11,12}$ parametrization. The hard-sphere diameter $d$ is chosen so as to minimize the free energy, ${ }^{5}$ which results in the condition

$$
\rho \int d \mathbf{r}\left[g_{000}^{00}(r)-g_{\mathrm{HS}}(r ; d)\right] \frac{\partial B_{\mathrm{HS}}(r ; d)}{\partial d}=0,
$$

where $g_{000}^{00}(r)$ is the center-to-center distribution function of the fluid and $g_{\mathrm{HS}}(r ; d)$ that of the reference hard-sphere system.
As noted in Ref. 1, the key to dealing with Eqs. (1) and (2) is the expansion of all angular functions in generalized spherical harmonics, i.e.,

$$
\begin{aligned}
X(12)= & \sum_{l_{1}, l_{2}, m, n_{1}, n_{2}}\left[\left(2 l_{1}+1\right)\left(2 l_{2}+1\right)\right]^{1 / 2} \\
& { }^{\times} X_{l_{1} l_{2} m}^{n_{1} n_{2}}\left(r_{12}\right) D_{m n_{1}}^{l_{1}}\left(\omega_{1}\right) * D_{\widetilde{m} n_{2}}^{l_{2}}\left(\omega_{2}\right)^{*},
\end{aligned}
$$

where, as in Ref. $1, \omega=(\phi, \theta, \chi)$ are the Euler angles referred to the axial line $\mathbf{r}_{12}$ between molecular centers and $D_{m n}^{l}(\omega)$ is a generalized spherical harmonic in the convention of Rose. ${ }^{13}$ One sees immediately that $g_{000}^{00}(r)$ in Eq. (3) is the first term in the expansion of $g(12)$.

Details of the algorithm used to solve Eqs. (1) and (2) in terms of the expansion coefficients of the functions involved can be found in Ref. 1. Once the set of nonlinear equations is solved we can proceed to calculate both thermodynamic and dielectric properties. Thus we have

$$
\frac{\beta U}{N}=2 \pi \rho \int_{0}^{\infty}\langle\beta u(12) g(12)\rangle_{\omega_{1} \omega_{2}} r_{12}^{2} d r_{12}
$$

and

$$
\frac{\beta P}{\rho}=1-\frac{2}{3} \pi \rho \int_{0}^{\infty}\left\langle r_{12} \frac{\partial \beta u(12)}{\partial r_{12}} g(12)\right\rangle_{\omega_{1} \omega_{2}} r_{12}^{2} d r_{12},
$$

where

$$
g(12)=\exp [-\beta u(12)+\gamma(12)+B(12)]
$$

and the angular brackets denote averages over all angles. In the expressions above, we have put as usual $\beta=1 / k_{B} T$, where $T$ is the absolute temperature and $k_{B}$ is Boltzmann's constant. Note that $g(12)$ is constructed indirectly from the summation of $\gamma(12)$ coefficients, rather than directly from those of $g$ (12) itself; this avoids the well-known poor convergence of the spherical harmonic series of $g(12)$ for anisotropic molecules. The reconstruction of $\gamma(12)$ from expansion (4) is known to be rapidly convergent and well behaved.

The atom-atom correlation functions are similarly obtained by integration over $g(12)$; in numerical format, we write

$$
\begin{aligned}
g_{\alpha \beta}(r)= & w^{3} \sum_{i=1}^{n_{r}} \sum_{i_{1}, i_{2}, j, k_{1}, k_{2}=1}^{n} w_{i_{1}} w_{i_{2}} g(12)\left(\frac{R_{i}}{r}\right)^{2} \\
& \times \Delta R \delta\left[r-\Re_{\alpha \beta}\left(R_{i}, x_{i_{1}}, x_{i_{2}}, y_{j}, z_{k_{1}}, z_{k_{2}}\right)\right],
\end{aligned}
$$

where $\Re_{\alpha \beta}\left(R_{i}, x_{i_{1}}, x_{i_{2}}, y_{j}, z_{k_{1}}, z_{k_{2}}\right)$ is the function that gives the atom-atom distance between sites $\alpha$ and $\beta$ for a given center-to-center separation $R_{i}$ and relative orientations described by $x_{i_{m}}=\cos \theta_{i_{m}}, y_{j}=\cos \phi_{j}$, and $z_{k_{m}}=\cos \chi_{k_{m}}$, where $\theta_{1}, \theta_{2}, \chi_{1}, \chi_{2}, \phi=\phi_{1}-\phi_{2}$ is the set of Euler angles required to describe the relative orientation of two nonlinear molecules. The $w_{i_{m}}$ in Eq. (8) are the weights of the GaussLegendre quadrature for the points $x_{i_{m}}$ and $w=1 / n$ is the constant weight of Gauss-Chebyshev quadrature for the points $y_{j}$ and $z_{k_{m}}$, with $n$ the number of grid points used in 
each angular integration and $n_{r}$ the number of grid points on the center-to-center $R$ axis, tabulated in equal size intervals $\Delta R$.

The dielectric constant can be obtained from the Kirkwood formula,

$$
\frac{(\epsilon-1)(2 \epsilon+1)}{9 \epsilon}=y g_{\mathrm{K}},
$$

where $y=4 \pi \beta \rho \mu^{2} / 9, \mu$ is the dipole moment, and $g_{\mathrm{K}}$ the Kirkwood $\mathrm{g}$ factor, a well-known order parameter that can be expressed in terms of correlation functions as

$$
g_{\mathrm{K}}=1+\frac{4}{3} \pi \rho \int_{0}^{\infty} r^{2} g(r ; 00,110) d r
$$

Here we have used the expansion coefficient $g(r ; 00,110)$ of $g(12)$ in the space-fixed reference frame. ${ }^{14}$

To incorporate the effects of polarizability by means of the SCMF approach, ${ }^{10}$ we recall that the effective dipole moment $\mu_{\text {eff }}$ is related to the permanent one $\mu$ by

$$
\beta \mu_{\mathrm{eff}}^{2}=\frac{\beta \mu^{2}}{(1+\alpha c)^{2}}+\frac{3 \alpha}{1+\alpha c},
$$

where $\alpha$ is the spherical polarizability ${ }^{15}$ and

$$
c=c\left(\mu_{\mathrm{eff}}\right)=\frac{2 U_{\mathrm{DD}}\left(\mu_{\mathrm{eff}}\right)+U_{\mathrm{DQ}}\left(\mu_{\mathrm{eff}}\right)}{N \mu_{\mathrm{eff}}^{2}} .
$$

Here $U_{\mathrm{DD}}$ and $U_{\mathrm{DQ}}$ are, respectively, the dipole-dipole and dipole-quadrupole contribution to the internal energy for a fluid with a dipole moment $\mu_{\text {eff }}$. These quantities can be easily evaluated once $g(12)$ is known by replacing $u(12)$ in Eq. (5) with its dipole-dipole and dipole-quadrupole terms. The standard procedure involves carrying out integral equation calculations for various values of $\mu_{\text {eff }}$ in the vicinity of the model dipole moment $\mu_{0}$, consistent with the initial charge distribution. One then plots $\mu_{\text {eff }}^{2}$ vs $\mu^{2}$, the latter obtained from Eqs. (11) and (12) (typically a linear relation), and from this graph interpolates the value of $\mu_{\text {eff }}^{2}$ that is consistent with the desired permanent dipole moment of the nonpolarizable system, $\mu_{0}$. The calculations are then repeated for this effective dipole moment and the dielectric constant is calculated from

$$
\frac{(\epsilon-1)(2 \epsilon+1)}{9 \epsilon}-\frac{\left(\epsilon_{\infty}-1\right)\left(2 \epsilon_{\infty}+1\right)}{9 \epsilon_{\infty}}=y_{\mathrm{eff}} g_{\mathrm{K}}\left(\mu_{\mathrm{eff}}\right),
$$

where $y_{\text {eff }}=4 \pi \beta \mu_{\text {eff }}^{2} \rho / 9$ and $\epsilon_{\infty}$ is the high-frequency dielectric constant, for which we use the Clausius-Mossotti value.

\section{RESULTS AND DISCUSSION}

The geometrical and potential energy parameters for the two models used to represent $\mathrm{SO}_{2}$ and for the point-charge model of $\mathrm{H}_{2} \mathrm{~S}$ are collected in Fig. 1 and Table I. Cross interactions are evaluated by means of the Lorentz-Berthelot mixing rules; charges are given in terms of the elementary electronic charge $e$. For $\mathrm{SO}_{2}$, we have studied a thermodynamic state corresponding to $T=249.5 \mathrm{~K}$ and $V=42.94$
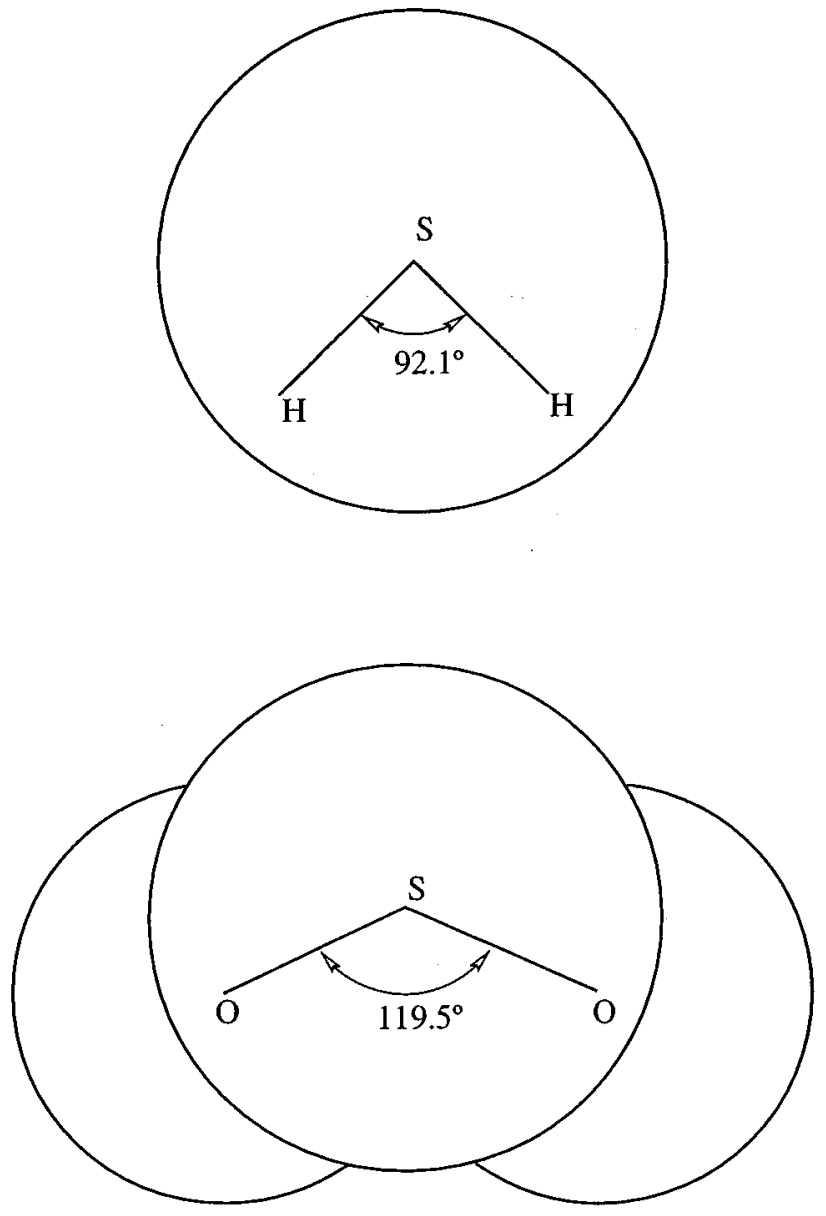

FIG. 1. Molecular geometries of $\mathrm{H}_{2} \mathrm{~S}$ (top) and $\mathrm{SO}_{2}$ (bottom) used in this work.

$\mathrm{cm}^{3}$ mole $^{-1}\left(\rho=0.0140 \AA^{-3}\right)$, and for $\mathrm{H}_{2} \mathrm{~S}, T=293 \mathrm{~K}$ and $V=43.10 \mathrm{~cm}^{3} \mathrm{~mole}^{-1}\left(\rho=0.0139 \AA^{-3}\right)$. We note that the charge distribution of model $\mathrm{B}$ for $\mathrm{SO}_{2}$ leads to a dipole moment $\mu_{0}=1.640 \mathrm{D}$, close to the experimental gas phase value $\mu_{\exp }=1.634 \mathrm{D}$, whereas the charge distribution in our model for $\mathrm{H}_{2} \mathrm{~S}$ leads to $\mu_{0}=2.087 \mathrm{D}$, which is more than double the experimental gas phase value of $\mu_{\exp }=0.974 \mathrm{D}$. Forester et ll $^{3}$ have proposed a more elaborate model that incorporates additional auxiliary charges and reduces considerably the net dipole moment while still producing reasonable results for the thermodynamic quantities. Nevertheless, to limit the complexity of the model while retaining the correct molecular shape, we will continue to use the model described in Table I. We will see later, however, that the artificially high dipole moment yields essentially meaningless dielectric properties.

With these considerations in mind, we have numerically solved the HNC and the optimized RHNC equations. In Fig. 2 we show the atom-atom pair distribution for model $\mathrm{A}$ of $\mathrm{SO}_{2}$. One sees that both the HNC and RHNC approximations yield good agreement with molecular dynamics results. ${ }^{16}$ Only in the $g_{\text {ss }}$ correlation is the HNC equation slightly out of phase, which is corrected by the RHNC equation. The 
TABLE I. Potential parameters for the molecular liquid models considered in this work.

\begin{tabular}{ccc}
\hline \hline & $\mathrm{SO}_{2}$ & \\
\hline Model A & Model B & $\mathrm{H}_{2} \mathrm{~S}$ \\
\hline$\varepsilon_{\mathrm{S}-\mathrm{S}}=182 \mathrm{~K} ; \varepsilon_{\mathrm{O}-\mathrm{O}}=72 \mathrm{~K}$ & $\varepsilon_{\mathrm{S}-\mathrm{S}}=154.5 \mathrm{~K} ; \varepsilon_{\mathrm{O}-\mathrm{O}}=62.3 \mathrm{~K}$ & $\varepsilon_{\mathrm{S}-\mathrm{S}}=125 \mathrm{~K}$ \\
$\sigma_{\mathrm{S}-\mathrm{S}}=3.61 \AA ; \sigma_{\mathrm{O}-\mathrm{O}}=3 \AA$ & $\sigma_{\mathrm{S}-\mathrm{S}}=3.585 \AA ; \sigma_{\mathrm{O}-\mathrm{O}}=2.993 \AA$ & $\sigma_{\mathrm{S}-\mathrm{S}}=3.71 \AA$ \\
& $q_{\mathrm{S}}=+0.470 \mathrm{e} ; q_{\mathrm{O}}=-0.235 \mathrm{e}$ & $q_{\mathrm{S}}=+0.470 \mathrm{e} ; q_{\mathrm{H}}=-0.235 \mathrm{e}$ \\
\hline \hline
\end{tabular}

latter approximation, however, tends to overestimate the first peak, probably due to the limitations of the spherically symmetric reference system. The same comments can be made about the results for model B, shown in Fig. 3. Thermodynamic results are given in Table II, specifically the internal energy and pressure for these models from both the HNC and RHNC approximations and from molecular dynamics (MD) simulations. We find that the internal energies are in

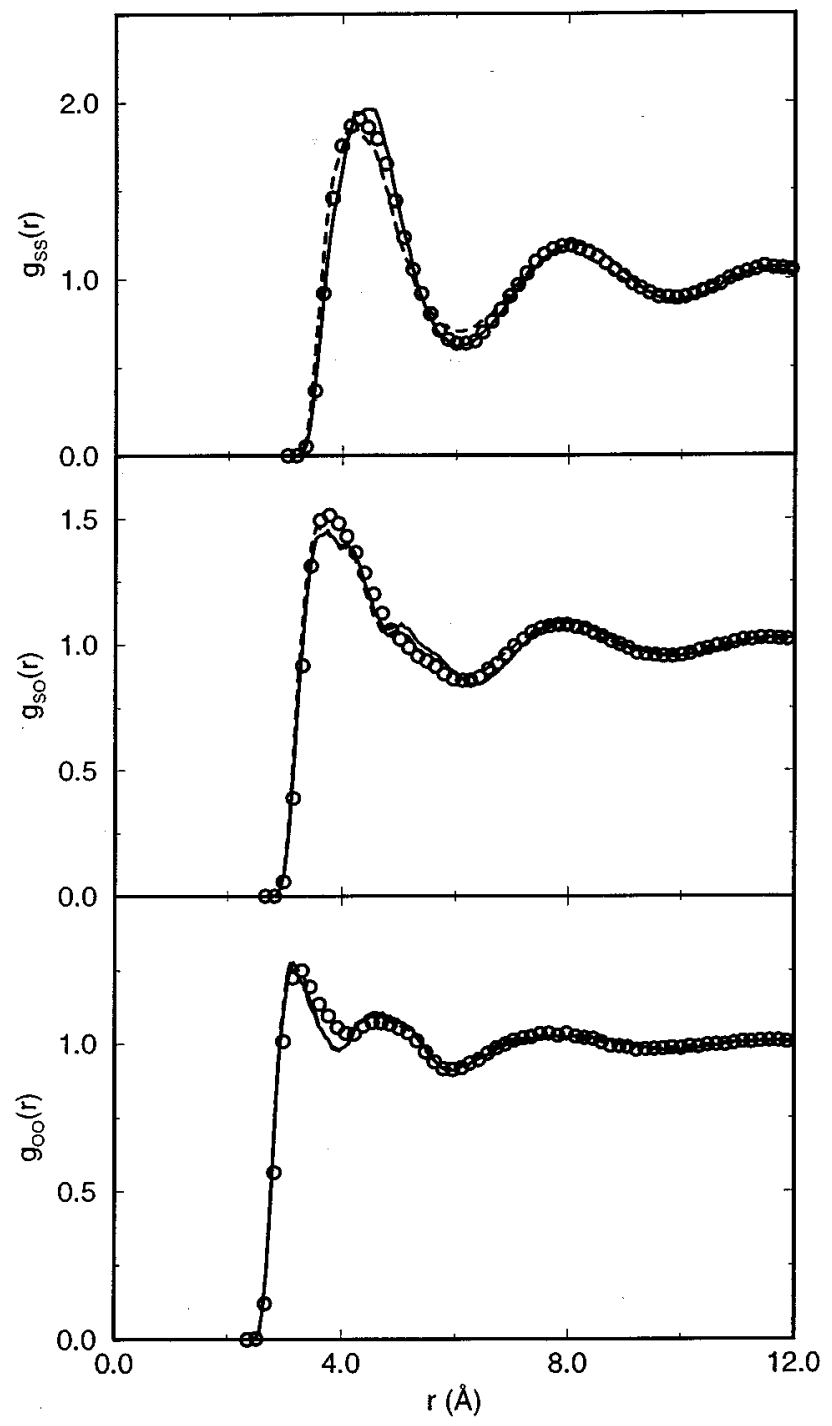

FIG. 2. Atom-atom distribution functions $g_{\alpha \beta}(r)$ for model A of liquid $\mathrm{SO}_{2}$ at $T=249.5 \mathrm{~K}$. Computed RHNC (continuous line) and HNC (dashed line) results vs MD simulation data (open circles). See Table I for model parameters. good agreement with MD for both approximations. For the compressibility factor, comparison with simulation shows that, as usual, the HNC equation overestimates this quantity, while here the improvement introduced by the spherically symmetric reference system falls short of what is needed. We have attempted to improve upon this by incorporating a nonspherical reference system with a bridge function evaluated using Verlet's modified approximation, along the lines explored in earlier works dealing with molecular fluids. ${ }^{17} \mathrm{Un}$ fortunately, the appearance of a pole in the denominator of

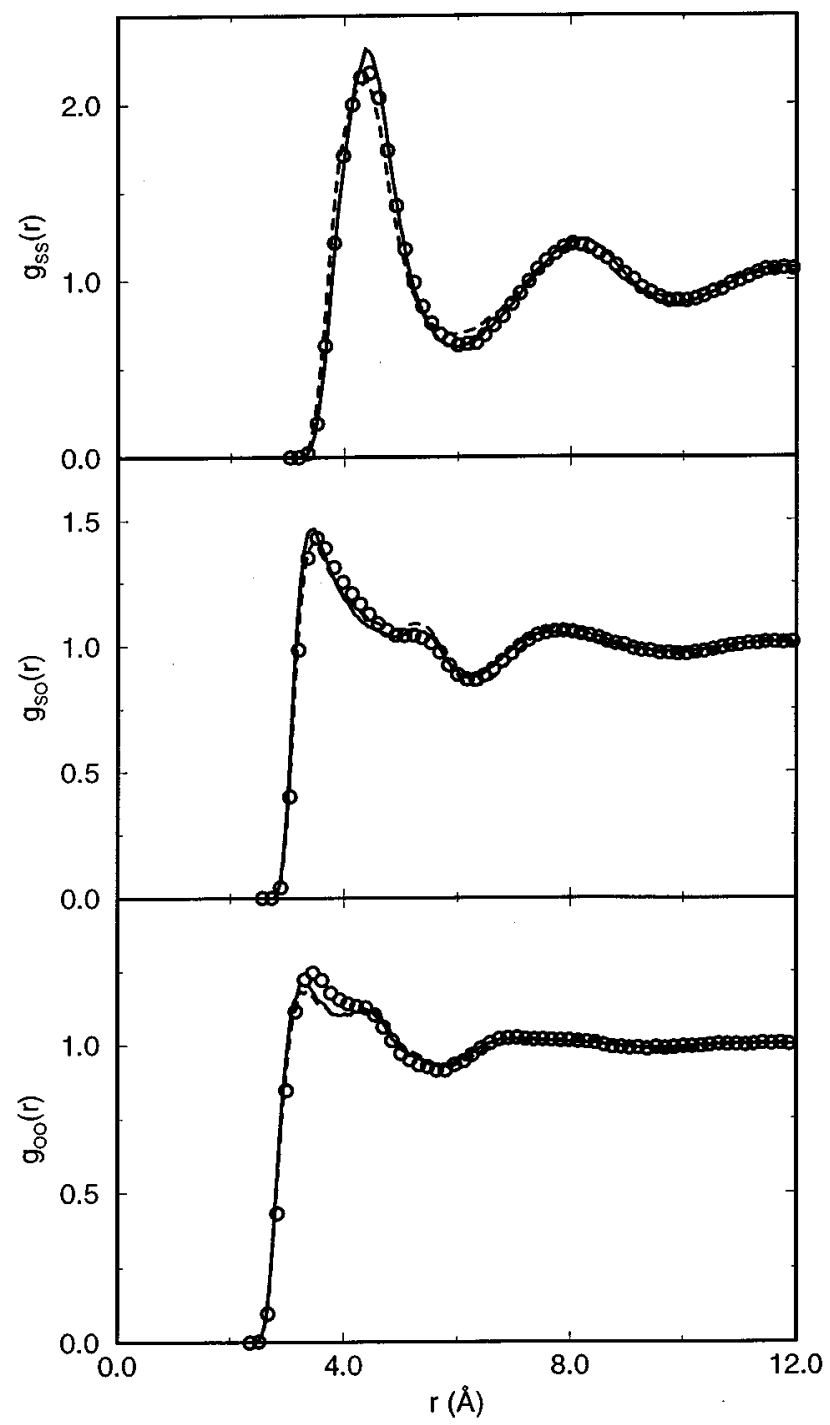

FIG. 3. Same as Fig. 2 for model $\mathrm{B}$ of liquid $\mathrm{SO}_{2}$. 
TABLE II. Internal energy and compressibility factor from theory and MD simulation, together with the experimental values taken from Refs. 2 and 3.

\begin{tabular}{lcccc}
\hline \hline & \multicolumn{3}{c}{$\mathrm{SO}_{2}$} & \multirow{2}{*}{$\mathrm{H}_{2} \mathrm{~S}$} \\
\cline { 2 - 4 } & Model A & \multicolumn{2}{c}{ Model B } \\
\hline$(\beta U / N)_{\text {exp. }}$ & & -11.51 & & -5.32 \\
$(\beta U / N)_{\mathrm{MD}}$ & $-11.55 \pm 0.03$ & & $-11.34 \pm 0.02$ & $-5.24 \pm 0.06$ \\
$(\beta U / N)_{\mathrm{HNC}}$ & -11.10 & & -11.20 & -5.12 \\
$(\beta U / N)_{\mathrm{RHNC}}$ & -11.26 & & -11.37 & -5.15 \\
$(\beta P / \rho)_{\mathrm{exp} .}$ & & 0.001 & & 0.18 \\
$(\beta P / \rho)_{\mathrm{MD}}$ & $0.1 \pm 0.2$ & & $0.2 \pm 0.4$ & $0.4 \pm 0.4$ \\
$(\beta P / \rho)_{\mathrm{HNC}}$ & 4.20 & & 3.81 & 1.05 \\
$(\beta P / \rho)_{\mathrm{RHNC}}$ & 2.60 & & 1.90 & 0.55 \\
\hline \hline
\end{tabular}

the Verlet bridge function prevents the calculation of this reference system for the cases of interest in this work. Thus improving upon the hard-sphere bridge function for the type of systems considered here remains an open question.

For liquid $\mathrm{H}_{2} \mathrm{~S}$, the smaller anisotropy of the molecule (see Fig. 1) improves the situation. The site-site distribution functions, depicted in Fig. 4, obtained from the HNC and RHNC equations are essentially indistinguishable and both are in excellent agreement with simulation. Table II shows that agreement of the calculated compressibility factor is better than that for $\mathrm{SO}_{2}$, especially for the RHNC result, while the internal energy values remain good.

If one is now willing to compare the computed dielectric constants with experimental values, the situation is quite different for the two substances. For liquid $\mathrm{SO}_{2}$ at the thermodynamic state specified earlier, the experimental value is $\epsilon_{\exp }=18.7$, while the theoretical estimates (without inclusion of polarizability) are $\epsilon_{\mathrm{HNC}}=10.0$ and $\epsilon_{\mathrm{RHNC}}=11.0$. An estimate from a 40000 step MD run gives $\epsilon_{\mathrm{MD}}=9.5 \pm 1.0$, in good agreement with these results. The differences between experimental and model results suggest that polarizability should play an important role here, which can be readily accepted by noting that the experimental value of the spherical polarizability is $\alpha \simeq 4.28 \AA^{-3}$. In contrast, the theoretical calculations for liquid $\mathrm{H}_{2} \mathrm{~S}$ yield $\epsilon_{\mathrm{HNC}}=16.8$ and $\epsilon_{\mathrm{RHNC}}=17.1$, which compare poorly with the experimental value of $\epsilon_{\mathrm{exp}} \simeq 6$. As mentioned earlier, this can be understood as a consequence of the anomalously high dipole moment (more than twice the experimental value) derived from our model charge distribution. Trying to incorporate polarizability into the present calculations for $\mathrm{H}_{2} \mathrm{~S}$ is not sensible.

For liquid $\mathrm{SO}_{2}$ we perform calculations at various effective dipole moments and thus, by means of Eqs. (11) and (12), obtain the representation of $\mu_{\text {eff }}^{2}$ vs $\mu^{2}$ shown in Fig. 5. The well-known linear form of this dependence makes it easy to extract the value of $\mu_{\text {eff }}$ that corresponds to $\mu_{0}=1.64$ $\mathrm{D} \quad\left(\mu^{*}=1.693\right)$; this turns out to be $\mu_{\mathrm{eff}}=1.88 \mathrm{D}$ $\left(\mu^{*}=1.946\right)$. These calculations have been performed in HNC approximation; the RHNC equation leads to very similar values of the effective dipole moments. With this $\mu_{\text {eff }}$, the new values of the dielectric constant obtained from Eq. (13) are $\epsilon_{\mathrm{HNC}}=18.0$ and $\epsilon_{\mathrm{RHNC}}=20.1$, in good agreement with the experimental $\epsilon_{\exp }=18.7$, as was the case earlier for $\mathrm{HCl}$ (Ref. 8) and $\mathrm{CH}_{3} \mathrm{CN}$ (Ref. 9). The SCMF calculation

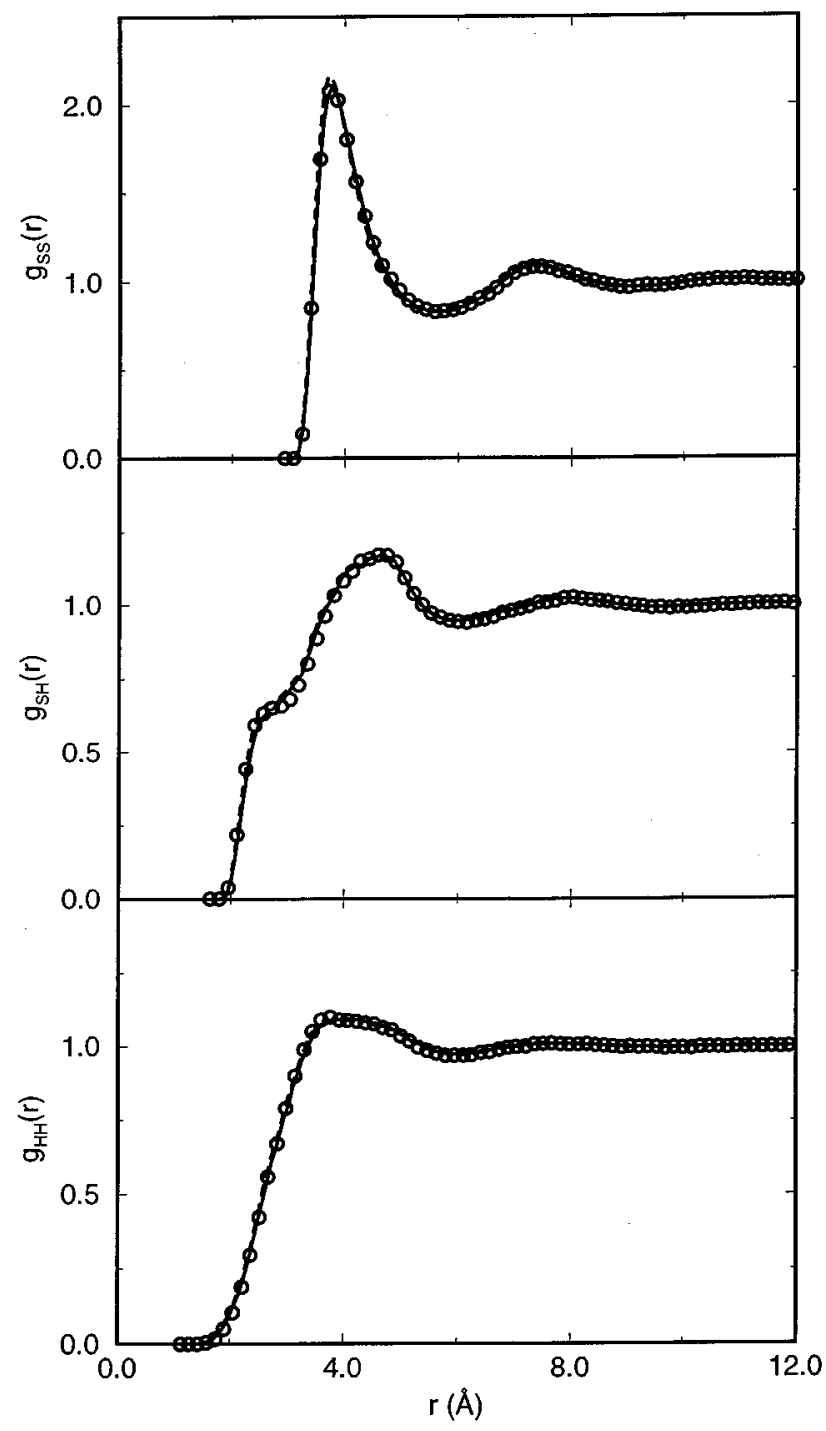

FIG. 4. Atom-atom distribution functions $g_{\alpha \beta}(r)$ for the model of liquid $\mathrm{H}_{2} \mathrm{~S}$ at $T=293 \mathrm{~K}$ used in this work. Computed RHNC (continuous line) and HNC (dashed line) results vs MD simulation data (open circles). Results for $g_{\alpha \beta}(r)$ from the two theoretical approximations are practically indistinguishable on the scale of the figure. See Table I for model parameters.

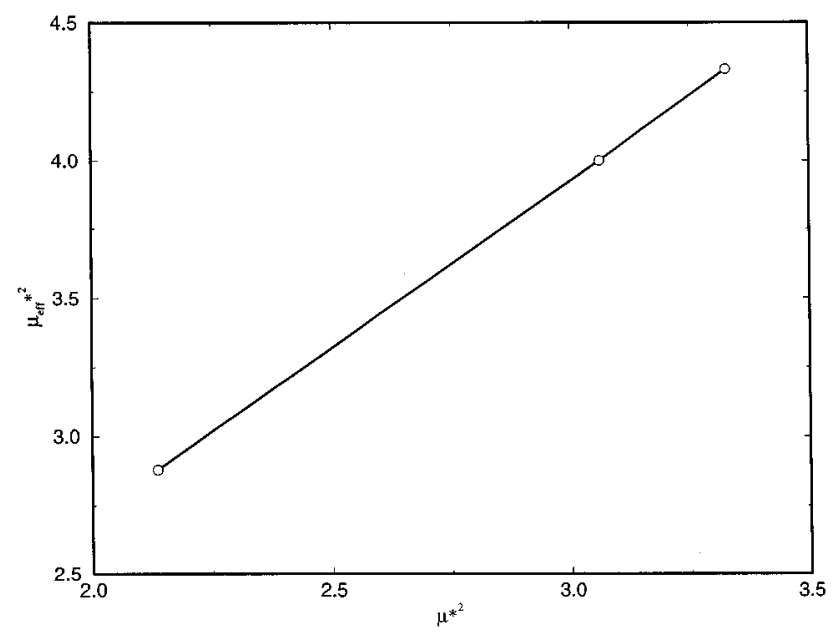

FIG. 5. Calculated values of $\mu_{\text {eff }}^{* 2}$ vs $\mu^{* 2}$ for model B of liquid $\mathrm{SO}_{2}$. The continuous line is merely a guide to the eye. 
combined with HNC or RHNC results is thus found to be a useful tool with remarkable predictive power so far as the dielectric constant is concerned.

\section{SUMMARY AND CONCLUSIONS}

In summary, we have presented another application of a powerful molecular integral equation implementation for the description of fluids composed of fully anisotropic particles with a $\mathrm{C}_{2 v}$ symmetry that is shared by numerous substances of interest. Computed results for both structure and thermodynamics are in good accord with molecular dynamics calculations. The use of a spherical reference system with the RHNC equation is particularly good for $\mathrm{H}_{2} \mathrm{~S}$, which is quite encouraging, given the similarities between the model used here to describe this substance and those used for liquid water consistent with the $\mathrm{C}_{2 v}$ symmetry, both with three (SPC) and four sites (TIP4P). Later work will be devoted to an extensive integral equation study of these rigid models of water.

The SCMF calculations carried out for liquid $\mathrm{SO}_{2}$ lead to dielectric constants that closely reproduce the experimental values. The model used for $\mathrm{H}_{2} \mathrm{~S}$, however, proves to be unsuitable for the calculation of dielectric properties, given its inadequate representation of the electrostatic components of the interactions.

\section{ACKNOWLEDGMENTS}

This research was financially supported by the Spanish Dirección General de Investigación Científica y Técnica
(DGICYT) under Grant No. PB94-0112. F.L. gratefully acknowledges sabbatical support from the Dirección General de Investigación y Desarrollo under Grant No. SAB95-0373.

${ }^{1}$ F. Lado, E. Lomba, and M. Lombardero, J. Chem. Phys. 103, 481 (1995).

${ }^{2}$ F. Sokolić, Y. Guissani, and B. Guillot, Mol. Phys. 56, 239 (1985).

${ }^{3}$ T. R . Forester, I. R. McDonald, and M. L. Klein, Chem. Phys. 129, 225 (1989).

${ }^{4}$ Y. Rosenfeld and N. W. Ashcroft, Phys. Rev. A 20, 1208 (1979).

${ }^{5}$ F. Lado, Phys. Lett. A 89, 196 (1982).

${ }^{6}$ M. Alvarez, E. Lomba, C. Martín, and M. Lombardero, J. Chem. Phys. 103, 3680 (1995).

${ }^{7}$ X. Ni and R. M. Fine, J. Phys. Chem. 96, 27 (1992).

${ }^{8}$ C. Martín, E. Lomba, M. Lombardero, F. Lado, and J. S. Høye, J. Chem. Phys. 100, 1599 (1994).

${ }^{9}$ J. A. Anta, E. Lomba, M. Alvarez, M. Lombardero, and C. Martín, J. Phys. Chem. B 101, 1451 (1997).

${ }^{10}$ S. L. Carnie and G. N. Patey, Mol. Phys. 47, 1129 (1982).

${ }^{11}$ L. Verlet and J. J. Weis, Phys. Rev. A 5, 939 (1972).

${ }^{12}$ E. W. Grundke and D. Henderson, Mol. Phys. 24, 269 (1972).

${ }^{13}$ M. E. Rose, Elementary Theory of Angular Momentum (Wiley, New York, 1957)

${ }^{14}$ See the discussion in Ref. 1 and F. Lado, Mol. Phys. 47, 283 (1982) regarding the spatial coefficients and axial coefficients.

${ }^{15}$ Explicit formulas for anisotropic polarizabilities can be found in J.S. Høye and E. Lomba, Mol. Phys. 81, 385 (1994).

${ }^{16}$ All MD results presented in this paper have been generated using the DL_POLY program. DL_POLY is a package of molecular simulation routines written by W. Smith and T. R. Forester. Copyright: The Engineering and Physical Sciences Research Council, acting through its Daresbury and Rutherford Appleton Laboratory at Daresbury Laboratory, 1994.

${ }^{17}$ M. Lombardero, C. Martín, and E. Lomba, J. Chem. Phys. 98, 6465 (1993). 